\title{
NUCLEAR MAGNETIC RESONANCE AND SPECTROPHOTOMETRIC STUDIES OF THE BINDING OF MUREXIDE WITH LITHIUM, SODIUM AND POTASSIUM IONS IN BINARY DIMETHYLSULFOXIDE-ACETONITRILE MIXTURES
}

\author{
Mojtaba Shamsipur* and Tayyebeh Madrakian \\ Department of Chemistry, Razi University, Kermanshah, Iran \\ <shamsipur@razi.ac.ir>
}

\begin{abstract}
The complexation reactions between murexide and $\mathrm{Li}^{+}, \mathrm{Na}^{+}$and $\mathrm{K}^{+}$ions in different dimethylsulfoxideacetonitrile mixtures were studied by both spectrophotometric and ${ }^{7} \mathrm{Li}-\mathrm{NMR}$ methods at $25^{\circ} \mathrm{C}$. The stoichiometry of the complexes was found to be $1: 1$. The formation constants of the resulting complexes, determined by both experimental techniques used, were found to be in satisfactory agreement. In all solvent mixtures studied, the stability of murexide complexes varied in the order $\mathrm{Na}>\mathrm{K}^{+}>\mathrm{Li}^{\top}$. There is an inverse relationship between the complex formation constants and amount of dimethylsulfoxide in the mixed solvent. A linear relationship was observed between $\log \mathrm{K}_{\mathrm{f}}$ for all complexes and the mole fraction of acetonitrile in solvent mixtures.
\end{abstract}

\section{Introduction}

Although the alkali and alkaline earth ions possess profound influences upon biological and materials sciences, ${ }^{1,2}$ their coordination chemistry, especially with classical ligands, has received very little attention, as compared to transition metal ions. ${ }^{3}$ Perhaps the most important reason for the delayed interest in this area of research is the quite weak interactions of conventional ligands with alkali and alkaline earth cations which are often beyond the sensitivities of most physicochemical techniques. However, due to the urgent need for understanding the vital role of cations like $\mathrm{Na}^{+}, \mathrm{K}^{+}, \mathrm{Mg}^{2+}$ and $\mathrm{Ca}^{2+}$ ions in biological systems, on the one hand, and the discovery of new macrocyclic ligands such as crown ethers ${ }^{4}$ and cryptands ${ }^{5}$ in the late 1960 's, on the other, a new era in the coordination chemistry of alkali and alkaline earth cations has opened during the past decades. . $^{68}$

Since, in the process of complex formation, the ligand must compete with solvent molecules for the cation, any variation in solvent properties is expected to change the apparent binding strength of the resulting complexes. Thus, an interesting possibility for understanding the nature of interactions between alkali and alkaline earth cations and conventional ligands in solution is the use of nonaqueous solvent of lower solvating abilities and dielectric constants than water.

Murexide, the ammonium salt of purpuric acid (I), has been frequently used as a suitable metallochromic indicator for a large number of metal ions over a wide range of experimental conditions. ${ }^{9-13}$ In most cases, the ligand forms 1:1 complexes with mono-di- and trivalent metal cations in aqueous, nonaqueous and mixed solvents. The metal binding of murexide is accompanied by a relatively strong shift of its absorption band, with $\lambda_{\max }=522 \mathrm{~nm}$ in aqueous solution, towards shorter wavelengths. Due to the very rapidly established equilibrium between metal ions and murexide in solution, ${ }^{14,15}$ the indicator has been successfully used in the competitive study of he thermodynamics ${ }^{16-20}$ and kinetics ${ }^{16.21-24}$ of a variety of metal ion complexes. We have previously reported the spectrophotometric study of murexide complexes with alkali, ${ }^{25}$ alkaline earth ${ }^{26}$ and transition metal ions ${ }^{27.8}$ in nonaqueous media. In this paper we report the complexation of $\mathrm{Li}^{+}, \mathrm{Na}^{+}$and $\mathrm{K}^{+}$ions in different binary dimethylsufoxide-acetonitrile mixtures by both nuclear magnetic resonance and spectrophotometry.

\section{Experimental}

Reagent grade murexide, lithium perchlorate, sodium perchlorate and potassium nitrate (all from Merck) were of the highest purity available and used without any further purification except vacuum drying over $\mathrm{P}_{2} \mathrm{O}_{5}$. Reagent grade acetonitrile (AN, Merck) and dimethylsulfoxide (DMSO, Merck) were purified and and dried by previously described methods. ${ }^{29}$ Different DMSO-AN solvent mixtures were prepared by weight.

All nuclear magnetic resonance measurements were made on a JEOL FX90Q FT-NMR Spectrometer with a field strength of $21.13 \mathrm{kG}$. At this field, lithium-7 resonates at $33.74 \mathrm{MHz}$. A 4.0 M aqueous $\mathrm{LiCl}$ solution was used as external reference and the reported lithium-7 chemical shifts refer to this solution. The paramagnetic (downfield) shift from the reference is designated as being positive. All chemical shift measurements were carried out at a probe temperature of $25.0 \pm 0.1^{\circ} \mathrm{C}$. 
Recording all electronic spectra and the absorbance measurements at fixed wavelengths were carried out on a Shimadzu model UV-263 spectrophotometer, equipped with a Shimadzu CPS controller model 260 thermostat at $25.0 \pm 0.1^{\circ} \mathrm{C}$.

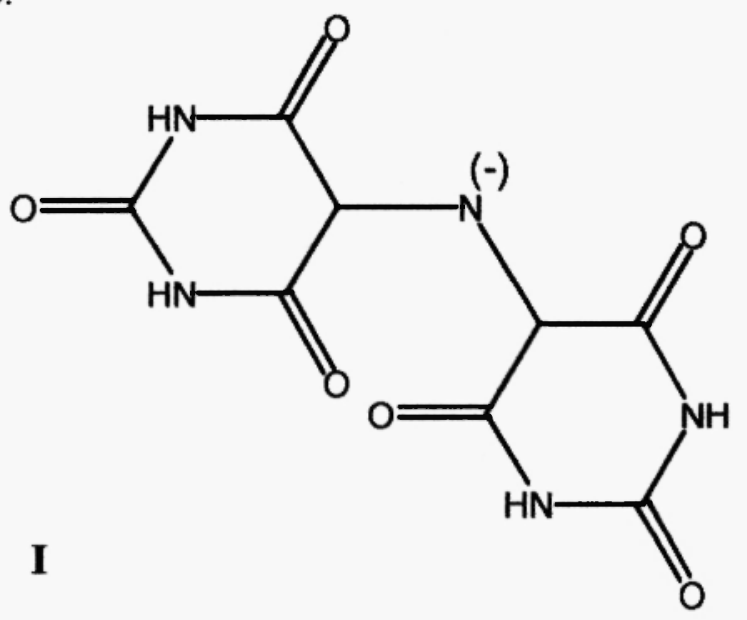

Results

Spectrophotometric Studies

The absorption spectra of murexide $\left(2.0 \times 10^{-5} \mathrm{M}\right)$ and its complexes with $\mathrm{Li}^{+}, \mathrm{Na}^{+}$and $\mathrm{K}^{+}$ion in various DMSO-AN mixtures were recorded. Sample visible spectra for titration of murexide with $\mathrm{Na}^{+}$ion in $7 \% \mathrm{AN}$ at $25^{\circ} \mathrm{C}$ are shown in Fig. 1. As it was pointed out before, ${ }^{10,27,30}$ all the resulting complexes are distinguished by a strong and ion specific blue shift $(-20-60 \mathrm{~nm})$, reasons of which are discussed elsewhere. ${ }^{25.26,31}$ The stoichiometry of the complexes in different DMSO-AN mixtures was examined by the method of continuous variations. ${ }^{32,33}$ Samples of the resulting plots are shown in Fig. 2 . In all cases, the metal ion to ligand mole ratio in the alkali-murexide complexes was found equal to 1:1. Moreover, the existence of a well-defined isosbestic point in the spectra of murexide upon titration with alkali cations (Fig. 1) is also a good indication for a simple 1:1 complexation equilibrium.

To determine the formation constants of the resulting $1: 1$ complexes in various DMSO-AN solutions, the spectra of solutions containing a constant amount of murexide $\left(2.0 \times 10^{-5} \mathrm{M}\right)$ and varying excess amounts of the alkali cations were obtained (cf. Fig. 1). The plots of $1 /\left(\varepsilon_{A}-\varepsilon_{M u}\right)$ vs. $1 / C_{M}$ gave straight lines, in accordance to equation (1): $:^{25.34}$

$$
\frac{1}{\varepsilon_{\mathrm{A}}-\varepsilon_{\mathrm{MMu}}}=\frac{1}{\varepsilon_{\mathrm{MMu}}-\varepsilon_{\mathrm{Mu}}}\left(1+\frac{1}{\overline{\mathrm{K}}_{\mathrm{f}} \tilde{\mathrm{C}}_{\mathrm{M}}}\right)
$$

where $\varepsilon_{A}=A / C_{M u}, A$ is the absorbance of the solution, $C_{M u}$ is the total concentration of alkali metal ion and $\varepsilon_{M u}$ and $\varepsilon_{M M u}$ are the molar absorptivities of the ligand and complex, respectively.

Sample linear plots of $1 /\left(\varepsilon_{\mathrm{A}}-\varepsilon_{\mathrm{Mu}}\right)$ vs. $1 / \mathrm{C}_{\mathrm{M}}$ for the murexide- $\mathrm{Li}^{+}$system in different DMSO-AN mixtures are shown in Fig. 3. The $K_{f}$ values evaluated from the slopes and intercepts of the resulting plots are summarized in Table $\mathrm{I}$.

\section{Nuclear Magnetic Resonance Studies}

Lithium-7 chemical shifts of $0.005 \mathrm{M}$ solutions of $\mathrm{Li}^{+}$ion in different DMSO-AN mixtures in the absence and presence of $0.005 \mathrm{M}$ of Na$^{+}$and $\mathrm{K}^{+}$ions were measured as a function of the mole ratio of murexide to lithium ion. In all cases, only one population-average resonance was observed, indicating that the exchange rate of the cation between the bulk solution and the complexed site is fast on the NMR time scale. The resulting mole ratio data are shown in Fig. 4. As it is seen, in all solvent mixtures, addition of murexide to the $\mathrm{Li}^{+}$ion solutions (curves number 1 in Fig. 4) causes an almost linear paramagnetic shift which begins to level off at mole ratios greater than unity. The slope of the corresponding mole ratio plots changes significantly at the point where the ligand-to-cation mole ratio is equal to one, emphasizing the formation of a relatively stable 1:1 complex. On the other hand, as it is obvious from Fig. 4 (curves number 2 and 3), unlike the case involved in the formation of $1: 1$ murexide- $\mathrm{Li}^{+}$complex in which the 
change in chemical shift with murexide/ $\mathrm{Li}^{+}$mole ratio is quite linear at mole ratios $<1$, the ${ }^{7} \mathrm{Li}$ chemical shift shows a curved relationship for murexide/ $/ i^{+}$mole ratios between 0 and 2 . However, in all cases, a plateau in chemical shift is reached at mole ratios greater than two. This is obviously indicative of the quantitative formation of 1:1 complexes of murexide with all cations used, which is in support of the results obtained spectrophotometrically.

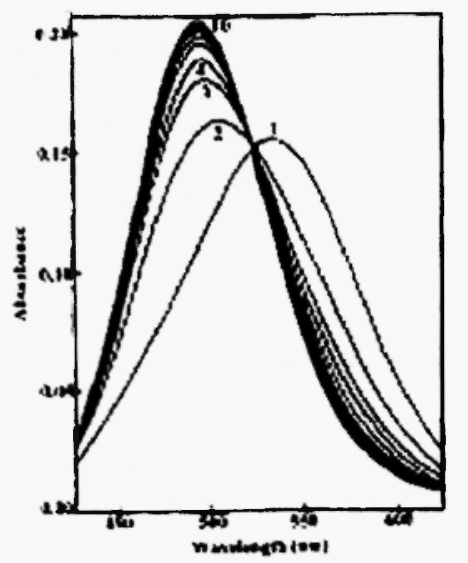

Fig. 1. Visible spectra for titration of murexide $\left(2.0 \times 10^{-3} \mathrm{M}\right)$ with $\mathrm{Na}^{+}$ion in $15 \% \mathrm{AN}$ at $25^{\circ} \mathrm{C}$.

Respective concentrations of the $\mathrm{Na}^{+}$ion $(\mathrm{M})$ in different solutions are: $1,0.0 ; 2,1.0 \times 10^{-5} ; 3,2.0 \times 10^{-3} ; 4$, $3.0 \times 10^{-3} ; 5,4.0 \times 10^{-3} ; 6,5.0 \times 10^{-3} ; 7,6.0 \times 10^{-3} ; 8,7.0 \times 10^{-3} ; 9,8.0 \times 10^{-3} ; 10,9.0 \times 10^{-3}$.

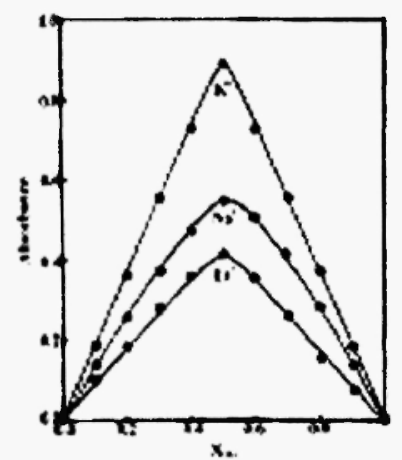

Fig. 2. Continuous variations plots for different $\mathrm{M}^{\dagger}$-murexide systems in DMSO solution.

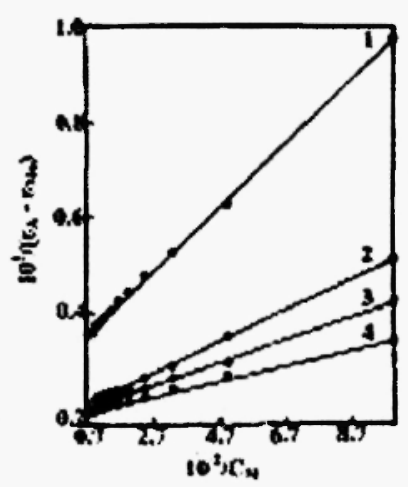

Fig. 3. Linear plots of $1 /\left(\varepsilon_{A}-\varepsilon_{M u}\right)$ vs. $1 / \mathrm{C}_{\mathrm{M}}$ for $\mathrm{Li}^{+}$-murexide in different solvent mixtures: (1) $0 \% \mathrm{AN}$; (2) $7 \%$ AN; (3) $15 \%$ AN; (4) $23 \%$ AN. 


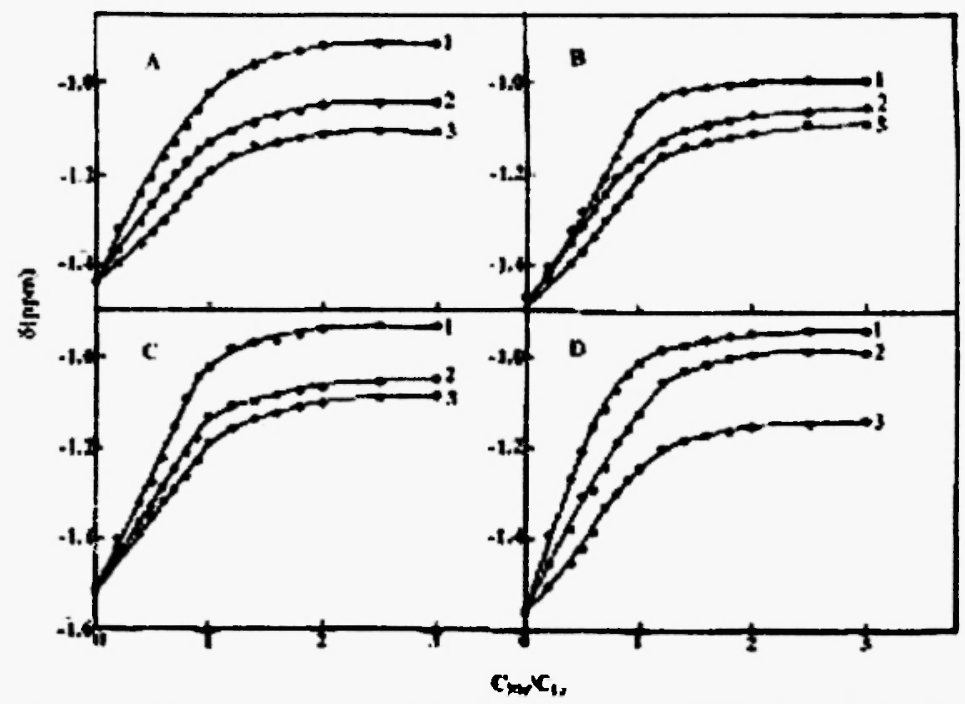

Fig. 4. Lithium-7 chemical shifts as a function of murexide/ $\mathrm{Li}^{+}$mole ratio in the absence (1) and presence of $\mathrm{K}^{+}$(2) and $\mathrm{Na}^{+}$(3) ions in different solvent mixtures: (A) $0 \% \mathrm{AN}$; (B) $7 \% \mathrm{AN}$; (C) $15 \% \mathrm{AN}$; (D) $23 \%$ AN.

TABLE I. Fornation constants of the murexide- $\mathrm{Li}^{+},-\mathrm{Na}^{+}$and $-\mathrm{K}^{+}$complexes in different DMSO-AN mixtures at $25^{\circ} \mathrm{C}^{\mathrm{a}}$

\begin{tabular}{lllll}
\hline \multirow{2}{*}{$\begin{array}{llll}* \\
\mathrm{~K}^{+}\end{array}$} & Method & \multicolumn{3}{c}{$\log \mathrm{K}_{\mathrm{f}}$} \\
\cline { 3 - 5 } & & & $\mathrm{Li}^{+}$ & $\mathrm{Na}^{+}$ \\
\hline 0 & NMR & 2.15 & 2.47 & 2.19 \\
& Spect. & 2.05 & 2.55 & 2.25 \\
& & $1.99^{\mathrm{b}}$ & $2.58^{\mathrm{b}}$ & $2.18^{\mathrm{b}}$ \\
7 & NMR & 2.41 & 2.75 & 2.53 \\
& Spect. & 2.37 & 2.72 & 2.51 \\
15 & NMR & 2.68 & 2.91 & 2.76 \\
& Spect. & 2.58 & 2.84 & 2.79 \\
23 & NMR & 2.82 & 3.09 & 2.92 \\
& Spect. & 2.81 & 3.13 & 2.98 \\
& & & &
\end{tabular}

SD on $\log \mathrm{K}_{\mathrm{f}}$ values is at the most \pm 0.05 .

Data taken from Ref. 25.

The formation constants of $1: 1$ murexide- $\mathrm{Li}^{+}$complex in different solvent mixtures were calculated from the variation of the ${ }^{7} \mathrm{Li}$ chemical shift with the murexide/ $\mathrm{Li}^{+}$mole ratio. It has been shown previously that the observed chemical shift is given by: ${ }^{35}$

$$
\begin{aligned}
\delta_{o b s}= & \left\{\left[K_{f} C_{M}-K_{f} C_{M u}-1\right)+\left(K_{f}^{2} C_{M u}{ }^{2}+K_{f} C_{M}{ }^{2}-2 K_{f}{ }^{i} C_{M u} C_{M}+\right.\right. \\
& \left.\left.\left.2 K_{f} C_{M u}+2 K_{f}+2 K_{f} C_{M}+1\right)^{i t}\right]\left(\delta_{M}-\delta_{M M u}\right) / 2 K_{f} C_{M}\right\}+\delta_{M M u}
\end{aligned}
$$

where $\delta_{M}$ and $\delta_{M M u}$ are the respective chemical shifts of the free and complexed lithium ion. A non-linear least-squares curve fitting program $K{ } N_{F I T}{ }^{36}$ was used to evaluate $K_{f}$ and $\delta_{M M u}$ values for the resulting complex, by fitting the $\delta_{\text {obs, }}, v \bar{s}$. $C_{M u}$ data (at constant $C_{L j}=0.005 \mathrm{M}$ ). A sample computer fit of the ${ }^{7} \mathrm{~L} i$ 
chemical shift-mole ratio data is shown in Fig. $5 \mathrm{~A}$, and the calculated $\log \mathrm{K}_{\mathrm{f}}$ values are also given in Table I. A fair agreement between the observed and calculated chemical shifts shown in Fig. 5A further the formation of a complex with 1:1 stoichiometry between $\mathrm{Li}^{+}$and murexide

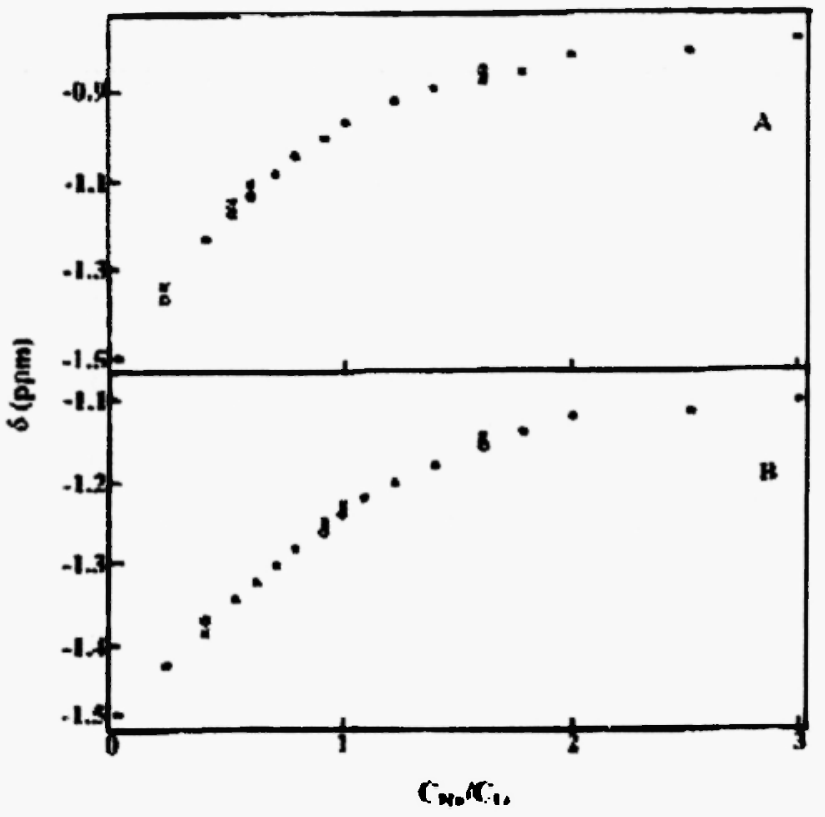

Fig. 5. Computer fit of lithium-7 chemical shift vs. muexide $/ \mathrm{Li}^{+}$mole ratio in $23 \% \mathrm{AN}$ at $25{ }^{\circ} \mathrm{C}$ in the anbsence (A) and presence (B) of $\mathrm{Na}^{+}$ions: $(x)$ experimental point; (o) calculated point; $(=)$ experimental and calculated points are the same within the resolution of the plots.

The competitive complexation equilibria for the case of $1: 1$ complexation between murexide and $\mathrm{Li}^{+}$ ions (i.e. $\mathrm{Na}^{+}$and $\mathrm{K}^{+}$ions) can be written as:

$$
\begin{array}{ll}
\mathrm{Li}+\mathrm{Mu}=\mathrm{Li}-\mathrm{Mu} & \mathrm{K}_{\mathrm{f}}=[\mathrm{Li}-\mathrm{Mu}] /[\mathrm{Li}][\mathrm{Mu}] \\
\mathrm{M}+\mathrm{Mu}=\mathrm{M}-\mathrm{Mu} & \mathrm{K}_{\mathrm{f}}^{\prime}=[\mathrm{M}-\mathrm{Mu}] /[\mathrm{M}][\mathrm{Mu}]
\end{array}
$$

The charges are omitted for simplicity. The free murexide concentration, [Mu], is then obtained from equation (5):

$$
\begin{aligned}
& K_{f} K_{f}[M u]^{3}-\left\{K_{i} K_{f}^{\prime}\left(C_{M u}-C_{L i}-C_{M}\right)-K_{f}-K_{f}^{\prime}\right\}[M u]^{2}-\left\{K_{f}\left(C_{M u}-C_{L i}\right)\right. \\
& \left.+K_{f}\left(C_{M u}-C_{M}\right)-1\right\}[M u]-C_{M u}=0
\end{aligned}
$$

In this case, the observed ${ }^{7} \mathrm{Li}$ chemical shift is obtained from equation (6): $:^{34}$

$$
\delta_{\text {obs }}=P_{L_{1}} \delta_{L_{1}}+P_{L_{1} M u} \delta_{\text {LiMu }}
$$

where $P_{\mathrm{Li}}$ and $\mathrm{P}_{\mathrm{LiMu}}$ are the mole fractions of free and complexed $\mathrm{Li}^{+}$ion, respectively. By substitution from equation (3) and the mass balance equation $\mathrm{C}_{\mathrm{Li}}=\left[\mathrm{Li}^{+}\right]+\left[\mathrm{Li}^{+}-\mathrm{Mu}\right]$, equation (6) can be written as:

$$
\delta_{o b s}=\left\{\delta_{\mathrm{Li}}+\delta_{\mathrm{LiMu}}[\mathrm{Mu}] \mathrm{K}_{\mathrm{f}}\right\} /\left\{1+\mathrm{K}_{\mathrm{f}}[\mathrm{Mu}]\right\}
$$

The $\mathrm{K}_{\mathrm{f}}$ values were evaluated by obtaining the free murexide concentration, [Mu], from equation (5) and fitting the chemical shift-mole ratio data to equation (7), by using the KINFIT program. 
A sample computer fit of the mole ratio data for a ternary $\mathrm{Li}^{+}-\mathrm{Mu}-\mathrm{K}^{+}$system is shown in Fig. $5 \mathrm{~B}$ and the resulting $\mathrm{K}_{f}$ values for murexide complexes with $\mathrm{Na}^{+}$and $\mathrm{K}^{+}$complexes are also included in Table $\mathrm{I}$. Our assumption of $1: 1$ stoichiometry of both $\mathrm{Li}^{+}$-murexide and $\mathrm{M}^{+}$-murexide complexes seem reasonable in the light of fair agreement between the observed and calculated chemical shifts.

\section{Discussion}

From the data given in Table $\mathrm{I}$, it is immediately obvious that, in the case of all metal ion-murexide complexes in different DMSO-AN mixtures, the $\mathrm{K}_{\mathrm{f}}$ values obtained with both NMR and spectrophotometric techniques (and those previously reported in pure DMSO) are in satisfactory agreement. It is also seen that, in all solvent mixtures used, the stability of the complexes vary in the order $\mathrm{Na}^{+}>\mathrm{K}^{+}>\mathrm{Li}^{+} . \mathrm{A}$ similar order has already been reported in the literature. ${ }^{25}$

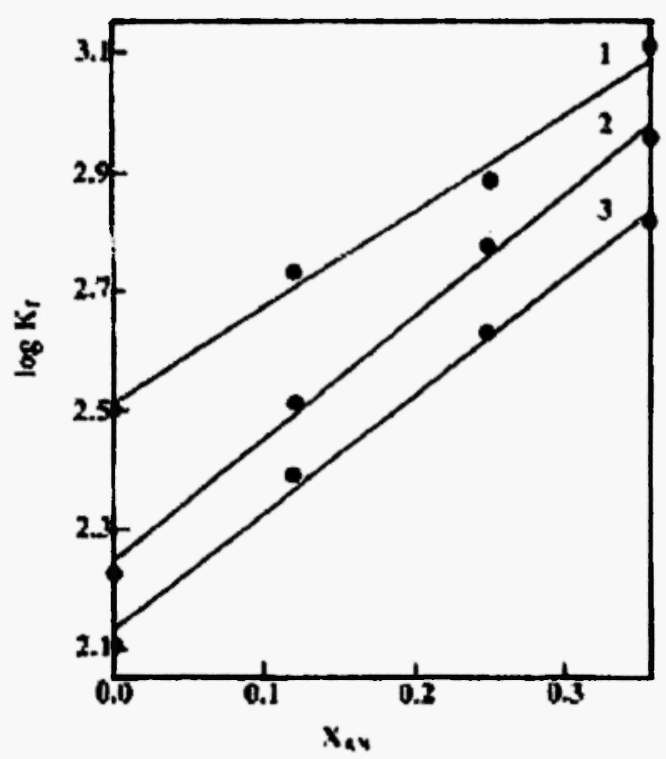

Fig. 6. Variation of average $\log \mathrm{K}_{\mathrm{f}}$ values for different murexide complexes with $\mathrm{X}_{\mathrm{AN}}$ : (1) $\mathrm{Na}^{+}$; (2) $\mathrm{K}^{+}$; (3) $\mathrm{Li}^{+}$.

The reported crystalline structures for the $\mathrm{Li}^{+37}$ and $\mathrm{K}^{+38}$ complexes of murexide show that the two approximately planar barbiturate rings of the ligand are not coplanar, the interaction between neighboring carbonyl groups causing torsion about the central nitrogen-carbon bonds. Thus, the highest stability observed for $\mathrm{Na}^{+}$complex among the alkali ions used is probably due to the proper ionic size of this cation, which could favor a suitable spatial fit with the flexible donating atoms of murexide in solution (i.e. the bridging nitrogen atom and neighboring oxygens). However, it should be noted that the thermodynamic stability is not just a measure of the absolute metal ion-ligand interaction, but is a measure of relative strength as compared to ionic solvation of all charged species involved in the complexation reaction (i.e. metal ion and murexide). Thus, the highest stability observed for the sodium-murexide complex results from a balance between the binding and solvation energies.

The data given in Table I clearly revealed that the solvent properties play a fundamental role in the complex formation processes. In all cases, the stability of resulting 1:1 complexes increases with increasing amount of AN in the mixed solvent. It is well known that the Gutmann donating ability of solvent, DN, ${ }^{39}$ plays a key role in different complexation reactions. ${ }^{6,7,19,20,25,26,28}$ DMSO is a solvent of high solvating ability $(\mathrm{DN}=29.8)$ which can strongly compete with murexide for the metal ions. Thus. it is not surprising that addition of $\mathrm{AN}$ as a solvent of relatively low donor number $(\mathrm{DN}=14.1)$ to $\mathrm{DMSO}$ will increase the stability of murexide complexes. It should be noted that the somewhat lover dielectric constant of AN (38.0) in comparison with that of DMSO (45.0) would also cause the electrostatic contribution to the bond formation to increase with increasing amount of $\mathrm{AN}$ in the solvent mixture.

It is interesting to note that there is actually a linear relationship between $\log \mathrm{K}_{\mathrm{f}}$ of the murexide complexes and the mole fraction of $\mathrm{AN}\left(\mathrm{X}_{\mathrm{AN}}\right)$ in the mixed solvent (Fig. 6). The same trend has already 
been reported for various complexes in different mixtures. preferential solvation of cations by DMSO is mainly responsible for such a monotonic dependence of the overall stability of the murexide complexes on the solvent composition.

\section{Referenees}

1. R. J. P. Williams, Q. Rev. Chem. Soc. 24, 331 (1970).

2. B. Dietrich, J. Chem. Educ. 62, 954 (1985).

3. N. S. Poonia and A. V. Bajaj, Chem. Rev. 79, 389 (1979).

4. C. J. Pedersen, J. Am. Chem. Soc. 89, 7017 (1967).

5. B. Dietrich, J. M. Lehn and J. P. Sauvage, Tetrahedron Lett. 2885 (1969).

6. R. M. Izatt, J. S. Bradshaw, S. A. Nielsen, J. D. Lamb, J. J. Christensen and D. Sen, Chem. Rev. 85, $271(1985)$.

7. R. M. Izatt, J. S. Bradshaw, K. Pawlak, R. L. Bruening and B. J. Tarbet, Chem. Rev. 92, 1261 (1992).

8. R. M. Izatt, K. Pawlak, J. S. Bradshaw and R. L. Bruening, Chem. Rev. 95, 2529 (1995).

9. A. Ringbom, Complexation in Analytical Chemistry (Interscience, New York, 1963).

10. G. Geier, Helv. Chim. Acta 50, 1879 (1967).

11. K. S. Balaji, S. Dinesh Kumar and P. Gupta-Bhaya, Anal. Chem. 50, 1972 (1978).

12. M. Fischer, W. Knoche, B. H. Robinson and J. H. Maclagan Wedderburn, J. Chem. Soc., Faraday I 75, 119 (1979).

13. C. D’Mara, J. Walsh and M. J. Hynes, Inorg. Chim. Acta 92, LI (1984).

14. G. Geier, Helv. Chim. Acta 51, 94 (1968).

15. H. Diebler, M. Eigen, G. Ilgenfritz, G. Maass and Winkler, Pure Appl. Chem. 20, 93 (1969).

16. V. M. Loyola, R. Pizer and R. G. Wilkins, J. Am. Chem. Soc 99, 7185 (1977).

17. H. Parham and M. Shamsipur, Polyhedron 11, 987 (1992).

18. N. Alizadeh and M. Shamsipur, Talanta 40, 503 (1993).

19. J. Ghasemi and M. Shamsipur, J. Coord. Chem. 31, 265 (1994).

20. H. Khajehsharifi and M. Shamsipur, J. Coord. Chem. 35, 289 (1995)

21. V. M. Loyola, R. G. Wilkins and R. Pizer, J. Am. Chem. Soc 97, 7382 (1475).

22. R. Pizer and R. Selzer, Inorg. Chem. 22, 1359 (1983).

23. J. Ghasemi and M. Shamsipur, Polyhedron 115, 923 (1996).

24. G. Azimi and M. Shamsipur, J. Coord. Chem. 47, 581 (1999).

25. M. Shamsipur, S. Madaeni and S. Kashanian, Talanta 36, 773 (1989).

26. S. Kashanian, M. B. Gholivand, S. Madaeni, A. Nikrahi and M. Shamsipur, Polyhedron 14, 1227 (1988).

27. M. Shamsipur and N. Alizadeh, Talanta 39, 1209 (1992).

28. J. Ghasemi and M. Shamsipur, J. Coord. Chem. 36, 183 (1995).

29. M. S. Greenberg and A. I. Popov, Spectrochim. Acta 31A, 697 (1975).

30. M. R. Fat'hi and M. Shamsipur, Spectrosc. Lett. 26, 1797 (1993).

31. R. Winkler, Struct. Bonding (Berlin) 10, 1 (1972).

32. P. Job, Ann. Chim. (Paris) 9, 113 (1928).

33. W. Likussar and D. F. Boltz, Anal. Chem. 43, 1262 (1971).

34. J. P. Birk, P. B. Chock and J. Halpern, J. Am. Chem. Soc. 90, 6959 (1968).

35. E. T. Roach, P. R. Handy and A. I. Popov, Inorg. Nucl. Chem. Lett. 9, 359 (1973).

36. V. A. Nicely and J. L. Dye, J. Chem. Educ. 48, 443 (1971).

37. H. B. Burgi, S. Djuric, M. Dobler and J. D. Dunitz, Helv. Chim. Acta 55, 1771 (1972).

38. R. L. Martin, A. H. White and A. C. Willis, J. Chem. Soc. Dalton Trans. 1336 (1977).

39. V. Gutmann, Coordination Chemistry in Nonaqueous solvents (Springer-Verlag, Vienna, 1968).

40. M. B. Gholivand and M. Shamsipur, Inorg. Chim. Acta 121, 53 (1986).

41. H. Parham and M. Shamsipur, J. Electroanal. Chem. 314, 71 (1991).

42. M. Daeidi and M. Shamsipur, J. Coord. Chem. 22, 131 (1990).

\section{Received: February 23, 2000 - Accepted: January 8, 2001 -} Accepted in publishable format: April 17, 2001 
